\title{
Moments from Progressive Type-II Censored Data of Marshall-Olkin Exponential Distribution
}

\author{
Mukhtar M. Salah \\ Department of Mathematics, Al-Quds Open University, Palestine, \\ West-Bank, Salfit P.O.Box 37, \\ Email: mshelleh@qou.edu.
}

\begin{abstract}
Marshall-Olkin exponential distribution has been studied by Salah, et al. [6]. In this paper, we derive several recurrence relations satisfied by the single moments and product moments of progressive type-II censored order statistics from Marshall-Olkin exponential distribution. These relations may then be used to compute all means, variances and covariances of progressive type-II censored ordered statistics based on Marshall-Olkin exponential distribution for arbitrary censoring scheme.
\end{abstract}

Keywords: order statistics, Marshall-Olkin exponential distribution, single moments, product moments, progressive type-II censored sample, exponential distribution, half logistic distribution.

AMS Subject Classification: 62G30,62E99, 60E05.

\section{Introduction}

Censored sampling arises in a life-testing experiment when ever the experimenter doesn't observe (either intentionally or unintentionally) the failure times of all units placed on a life-test. For example consider a life-testing experiment where $n$ items are kept under observation, these items could be systems, computers, individuals in a clinical trial, in reliability study experiment, so that the removal of units from the experimentation is pre-planned and intentional, and is done in order to provide saving in terms of time and cost associated with testing. The data obtained from such experiments are called 
censored data. There are many types of censoring scheme, here we mention some of them, let us consider $n$ unites are placed on a life-test then, type-I (time) censoring: Suppose it is decided to terminate the experiment at a predetermined time $t$, so that only failure time of these items that failed prior to this time recorded, the data so obtained from this process constitute a type-I censored sample. Type-II censoring: If the experiment is terminated at the $r t h$ failure, that is at time $X_{r: n}$, we obtain type-II censored sample, here $r$ is fixed, while $X_{r: n}$ the duration of the experiment is random. Many articles in this literature have discussed inferential method under type-I and type-II censoring for various parametric families of distributions, for more details, see for example, Balakrishnan and Cohen [9], Pradhan and Kundu [2] and Sultan et al. [3].

A generalization of type-II censored sample is a progressive type-II right censoring: Suppose $n$ units taken from the same population are placed on a life test. At the first failure time of one of the $n$ units, a number $R_{1}$ of the surviving units is randomly withdrawn from the test, at the second failure time, another $R_{2}$ surviving units are selected at random and taken out of the experiment, and so on. Finally at the $m$ th failure, the remaining $R_{m}=$ $n-m-R_{1}-R_{2}-\ldots-R_{m-1}$ unit are removed. In this scheme $\left(R_{1}, R_{2}, \ldots, R_{m}\right)$ is pre-fixed. The resulting $m$ order failure times, which denote by

$$
X_{1: m: n}^{\left(R_{1}, R_{2}, \ldots, R_{m}\right)}, X_{2: m: n}^{\left(R_{1}, R_{2}, \ldots, R_{m}\right)}, \ldots, X_{m: m: n}^{\left(R_{1}, R_{2}, \ldots, R_{m}\right)},
$$

are referred to as progressive type-II right censored order statistics. The special case when $R_{1}=R_{2}=\ldots=R_{m-1}=0$, so that $R_{m}=n-m$ this scheme reduces to the conventional type-II censoring scheme, also when $R_{1}=R_{2}=$ $\ldots=R_{m}=0$, so that $m=n$, then no censoring happen ( complete data case). For more detailed discussion about progressive censoring, one may refer to Balakrishnan and Aggarwala [11]. If the failure times are based on an absolutely continuos distribution function $F$ with probability density function (pdf) $f$, the joint probability density function of the progressive censored failure times $X_{1: m: n}, X_{2: m: n}, \ldots, X_{m: m: n}$, is given by

$$
\begin{aligned}
f_{X_{1: m: n}, \ldots, X_{m: m: n}}\left(x_{1}, x_{2}, \ldots, x_{m}\right) & =A(n, m-1)_{i=1}^{m} f\left(x_{i}\right)\left[1-F\left(x_{i}\right)\right]^{R_{i}}, \\
-\infty & <x_{1}<x_{2}<\ldots<x_{m}<\infty
\end{aligned}
$$

where $f(),. F($.$) are, respectively, pdf and the cumulative distribution func-$ tion (cdf) of the random sample and

$A(n, m-1)=n\left(n-1-R_{1}\right)\left(n-2-R_{1}-R_{2}\right) \ldots\left(n-m+1-R_{1}-\ldots-R_{m-1}\right)$.

The purpose of this paper is to consider progressive type-II censored data from the Marshall-Olkin exponential distribution, for simplicity, denoted by 
MOE distribution. The cdf of MOE distribution is given as follows:

$$
F(x)=1-\frac{\alpha e^{-x}}{\left(1-(1-\alpha) e^{-x}\right)}, \quad 0 \leq x<\infty, \alpha>0 .
$$

And its pdf is given as follows:

$$
f(x)=\frac{\alpha e^{-x}}{\left(1-(1-\alpha) e^{-x}\right)^{2}}, \quad 0 \leq x<\infty, \alpha>0 .
$$

When $\alpha=1$, the MOE distribution reduces to the standard exponential distribution, and when $\alpha=2$, the MOE distribution reduces to the half logistic distribution. Note that the cdf and the pdf given in Eqs. (2) and (3) are respectively for the standard MOE distribution for more details see, Salah [5].

From Eqs.(2) and (3) one can be readily seen the following three relations

$$
\begin{gathered}
f(x)=[1-F(x)]+\frac{1-\alpha}{\alpha}[1-F(x)]^{2}, \alpha>0, \\
f(x)=F(x)[1-F(x)]+\frac{1}{\alpha}[1-F(x)]^{2}, \alpha>0, \\
f(x)=\frac{1}{\alpha}\left[1+(\alpha-2) F(x)+(1-\alpha) F^{2}(x)\right], \alpha>0 .
\end{gathered}
$$

We use these relations in the next sections in order to establish several recurrence relations satisfied by the single and the product moments of the progressive type-II right censored order statistics.

Rest of the paper is organized as follows: In Section 2, we derive several new recurrence relations satisfied by the single moments of progressive typeII right censored order statistics from the MOE distribution. In Section 3, we derive and establish some recurrence relations satisfied by the product moments of progressive type-II right censored order statistics from the MOE distribution. Finally, in Section 4, we show how the recursive computational used for computing the single and product moment in recursive algorithm.

\section{Recurrence Relations for Single Moments}

The moments of order statistics have been recursively derived see, Salah, et al. [6], for a complete sample. In this section, we establish several new recurrence relations satisfied by the single moments of progressive type-II right censored order statistics from the MOE distribution. These recurrence relations may be used to compute the means, variances and covariances of MOE progressive type-II right censored order statistics for all sample sizes $n$ and all censoring schemes $\left(R_{1}, R_{2}, \ldots, R_{m}\right), m \leq n$. 
Let $X_{1}, X_{2}, \ldots, X_{n}$ be a random sample from the standard MOE distribution with cdf and pdf given in Eqs. (2 ) and (3) respectively. The corresponding progressive type-II right censored order statistics with censoring scheme $\left(R_{1}, R_{2}, \ldots, R_{m}\right), m \leq n$ will be

$$
X_{1: m: n}^{\left(R_{1}, R_{2}, \ldots, R_{m}\right)}, X_{2: m: n}^{\left(R_{1}, R_{2}, \ldots, R_{m}\right)}, \ldots, X_{m: m: n}^{\left(R_{1}, R_{2}, \ldots, R_{m}\right)} .
$$

The single moments of the progressive type-II right censored order statistics from the MOE distribution can be written as follows see, Balakrishnan and Aggarwala, [11]

$$
\begin{aligned}
\mu_{i: m: n}^{\left(R_{1}, R_{2}, \ldots, R_{m}\right)^{(k)}}= & E\left[X_{i: m: n}^{\left(R_{1}, R_{2}, \ldots, R_{m}\right)^{(k)}}\right] \\
= & A(n, m-1) \int_{0<x_{1}<x_{2}<\ldots<x_{m}<\infty} x_{i}^{k} f\left(x_{1}\right)\left[1-F\left(x_{1}\right)\right]^{R_{1}} \\
& \times f\left(x_{2}\right)\left[1-F\left(x_{2}\right)\right]^{R_{2}} \ldots f\left(x_{m}\right)\left[1-F\left(x_{m}\right)\right]^{R_{m}} d x_{1} \ldots d x_{m},
\end{aligned}
$$

where $f($.$) and F($.$) are given respectively in Eqs. (2),(3), and A(n, m-1)$ as defined in Eq. (1). When $k=1$, the superscript in the notation of the mean of the progressive type-II right censored order statistics may be omitted without any confusion. The single moments of the progressive type-II right censored order statistics given in Eq. (7) satisfies the following recurrence relations.

Theorem 2.1 For $2 \leq m \leq n$ and $k \geq 0$, we have

$$
\begin{aligned}
\mu_{1: m: n+1}^{\left(R_{1}+1, R_{2}, \ldots, R_{m}\right)^{(k+1)}=} & \frac{(n+1) \alpha}{n(1-\alpha)\left(R_{1}+2\right)}\left[(k+1) \mu_{1: m: n}^{\left(R_{1}, \ldots, R_{m}\right)^{(k)}}-\left(R_{1}+1\right) \mu_{1: m: n}^{\left(R_{1}, \ldots, R_{m}\right)^{(k+1)}}\right. \\
& -\left(n-R_{1}-1\right) \mu_{1: m-1: n}^{\left.\left(R_{1}+R_{2}+1, R_{3}, \ldots, R_{m}\right)^{(k+1)}\right]} \\
& -\frac{\left(n-R_{1}-1\right)}{\left(R_{1}+2\right)} \mu_{1: m-1: n+1}^{\left(R_{1}+R_{2}+2, R_{3}, \ldots, R_{m}\right)^{(k+1)}} \\
\alpha> & 0, \alpha \neq 1 .
\end{aligned}
$$

Proof. From Eq.(7) and Eq.(4) we have

$$
\begin{aligned}
\mu_{1: m: n}^{\left(R_{1}, R_{2}, \ldots, R_{m}\right)^{(k)}=} & A(n, m-1) \int_{0<x_{2}<\ldots<x_{m}<\infty} L\left(x_{2}\right) f\left(x_{2}\right)\left[1-F\left(x_{2}\right)\right]^{R_{2}} \ldots \\
& \times f\left(x_{m}\right)\left[1-F\left(x_{m}\right)\right]^{R_{m}} d x_{2} \ldots d x_{m}
\end{aligned}
$$

where

$$
L\left(x_{2}\right)=\int_{0}^{x_{2}} x_{1}^{k}\left[1-F\left(x_{1}\right)\right]^{R_{1}+1} d x_{1}+\frac{1-\alpha}{\alpha} \int_{0}^{x_{2}} x_{1}^{k}\left[1-F\left(x_{1}\right)\right]^{R_{1}+2} d x_{1} .
$$


By integrating the right hand side of the last equation by parts, we obtain

$$
\begin{aligned}
L\left(x_{2}\right)= & \frac{x_{2}^{k+1}}{k+1}\left[1-F\left(x_{2}\right)\right]^{R_{2}+1}+\frac{R_{1}+1}{k+1} \int_{0}^{x_{2}} x_{1}^{k+1} f\left(x_{1}\right)\left[1-F\left(x_{1}\right)\right]^{R_{1}} d x_{1} \\
& +\frac{1-\alpha}{\alpha} \frac{x_{2}^{k+1}}{k+1}\left[1-F\left(x_{2}\right)\right]^{R_{2}+2}+\frac{\left(R_{1}+2\right)(1-\alpha)}{(k+1) \alpha} \int_{0}^{x_{2}} x_{1}^{k+1} f\left(x_{1}\right)\left[1-F\left(x_{1}\right)\right]^{R_{1}+1} d x_{1} .
\end{aligned}
$$

Substituting Eq. (10) in Eq. (9) and using the definition of the single moment given in Eq. (7) we simply have

$$
\begin{aligned}
\mu_{1: m: n}^{\left(R_{1}, R_{2}, \ldots, R_{m}\right)^{(k)}=} & \left(n-R_{1}-1\right) \frac{1}{k+1} \mu_{1: m-1: n}^{\left(R_{1}+R_{2}+1, \ldots, R_{m}\right)^{(k+1)}}+\frac{n\left(n-R_{1}-1\right)}{n+1} \frac{1-\alpha}{(k+1) \alpha} \\
& \times \mu_{1: m-1: n+1}^{\left(R_{1}+R_{2}+2, \ldots, R_{m}\right)^{(k+1)}}+\frac{R_{1}+1}{k+1} \mu_{1: m: n}^{\left(R_{1}, R_{2}, \ldots, R_{m}\right)^{(k+1)}} \\
& +\frac{n\left(R_{1}+2\right)(1-\alpha)}{(n+1)(k+1) \alpha} \mu_{1: m: n+1}^{\left(R_{1}+1, \ldots, R_{m}\right)^{(k+1)}} .
\end{aligned}
$$

The result follows.

Corollary 2.1 For $\alpha=2$, we have

$$
\begin{aligned}
\mu_{1: m: n}^{\left(R_{1}, R_{2}, \ldots, R_{m}\right)^{(k)}=} & \frac{1}{k+1}\left[\left(R_{1}+1\right) \mu_{1: m: n}^{\left(R_{1}, R_{2}, \ldots, R_{m}\right)^{(k+1)}}+\left(n-R_{1}-1\right) \mu_{1: m-1: n}^{\left(R_{1}+R_{2}+1, \ldots, R_{m}\right)^{(k+1)}}\right] \\
& -\frac{n}{2(n+1)(k+1)}\left[\left(n-R_{1}-1\right) \mu_{1: m-1: n+1}^{\left(R_{1}+R_{2}+2, \ldots, R_{m}\right)^{(k+1)}}\right. \\
& +\left(R_{1}+2\right) \mu_{1: m: n+1}^{\left.\left(R_{1}+1, R_{2}, \ldots, R_{m}\right)^{(k+1)}\right] .}
\end{aligned}
$$

Corollary 2.2 For $\alpha=1$, we have

$$
\mu_{1: m: n}^{\left(R_{1}, R_{2}, \ldots, R_{m}\right)^{(k+1)}}=\frac{n-R_{1}-1}{R_{1}+1} \mu_{1: m-1: n}^{\left(R_{1}+R_{2}+1, \ldots, R_{m}\right)^{(k+1)}}-\frac{k+1}{R_{1}+1} \mu_{1: m-1: n}^{\left(R_{1}, \ldots, R_{m}\right)^{(k)}} .
$$

Theorem 2.2 For $m=1, n=1,2, \ldots$, and $k \geq 0$, we have

$$
\mu_{1: 1: n+1}^{(n)^{(k+1)}}=\frac{\alpha}{1-\alpha}\left[\frac{k+1}{n} \mu_{1: 1: n}^{(n-1)^{(k)}}-\mu_{1: 1: n}^{(n-1)^{(k+1)}}\right], \alpha>0, \alpha \neq 1 .
$$

If $\alpha=1$, we have

$$
\mu_{1: 1: n}^{(n-1)^{(k+1)}}=\frac{k+1}{n} \mu_{1: 1: n}^{(n-1)^{(k)}}
$$


Proof. Let $m=1, n=1,2, \ldots$, and $k \geq 0$. Using Eqs. (7) and (4) we obtain

$$
\mu_{1: 1: n}^{\left(R_{1}\right)^{(k+1)}}=n \int_{0}^{\infty} x_{1}^{k}\left[1-F\left(x_{1}\right)\right]^{R_{1}+1} d x_{1}+n \frac{\alpha}{1-\alpha} \int_{0}^{\infty} x_{1}^{k}\left[1-F\left(x_{1}\right)\right]^{R_{1}+2} d x_{1} .
$$

Integrating the last equation by parts, to get

$$
\mu_{1: 1: n}^{\left(R_{1}\right)^{(k)}}=\frac{n}{k+1} \mu_{1: 1: n}^{\left(R_{1}\right)^{(k+1)}}+\frac{1-\alpha}{\alpha} \frac{n}{k+1} \mu_{1: 1: n+1}^{\left(R_{1}+1\right)^{(k+1)}} .
$$

The results in Eqs. (11) and (12) follow directly from Eq.(13).

Theorem 2.3 For $2 \leq i \leq m-1, m \leq n$ and $k \geq 0$ we have

$$
\begin{aligned}
\mu_{i: m: n+1}^{\left(R_{1}, \ldots, R_{i}+1, \ldots, R_{m}\right)^{(k+1)}=} & \frac{\alpha}{1-\alpha} \frac{1}{R_{i}+2} \frac{A(n+1, i-1)}{A(n, i-1)}\left[(k+1) \mu_{i: m: n}^{\left(R_{1}, R_{2}, \ldots, R_{m}\right)^{(k)}}\right. \\
& +\left(n-R_{1}-\ldots-R_{i-1}-i+1\right) \mu_{i-1: m-1: n}^{\left(R_{1}, \ldots, R_{i-2}, R_{i-1}+R_{i}+1, R_{i+1} \ldots, R_{m}\right)^{(k+1)}} \\
& -\left(R_{i}+1\right) \mu_{i: m: n}^{\left(R_{1}, R_{2}, \ldots, R_{m}\right)^{(k+1)}} \\
& -\left(n-R_{1}-\ldots-R_{i}-i\right) \mu_{i: m-1: n}^{\left(R_{1}, \ldots, R_{i-1}, R_{i}+R_{i+1}+1, R_{i+2} \ldots, R_{m}\right)^{(k+1)}} \\
& -\frac{1-\alpha}{\alpha} \frac{A(n, i)}{A(n+1, i-1)} \mu_{i: m-1: n+1}^{\left(R_{1}, \ldots, R_{i-1}, R_{i}+R_{i+1}+2, R_{i+2} \ldots, R_{m}\right)^{(k+1)}} \\
& \left.+\frac{1-\alpha}{\alpha} \frac{A(n, i-1)}{A(n+1, i-2)} \mu_{i-1: m-1: n+1}^{\left.\left(R_{1}, \ldots, R_{i-2}, R_{i-1}+R_{i}+2, R_{i+1} \ldots, R_{m}\right)^{(k+1)}\right]}\right] \\
\alpha> & 0, \alpha \neq 1 .
\end{aligned}
$$

Proof. From Eqs.(7) and (4) we may write

$$
\begin{aligned}
\mu_{i: m: n}^{\left(R_{1}, R_{2}, \ldots, R_{m}\right)^{(k)}=} & A(n, m-1)_{0<x_{1}<\ldots<x_{i-1}<x_{i+1}<\ldots<x_{m}} I\left(x_{i+1}\right) f\left(x_{1}\right)\left[1-F\left(x_{1}\right)\right]^{R_{1}} \\
& \times \ldots f\left(x_{i-1}\right)\left[1-F\left(x_{i-1}\right)\right]^{R_{i-1}} f\left(x_{i+1}\right)\left[1-F\left(x_{i+1}\right)\right]^{R_{i+1}} \\
& \times \ldots f\left(x_{m}\right)\left[1-F\left(x_{m}\right)\right]^{R_{m}} d x_{1} \ldots d x_{i-1} d x_{i+1} \ldots d x_{m}
\end{aligned}
$$

where

$$
I\left(x_{i+1}\right)=\int_{x_{i-1}}^{x_{i+1}}\left[x_{i}^{k}\left[1-F\left(x_{i}\right)\right]^{R_{i}+1}+\frac{1-\alpha}{\alpha} x_{i}^{k}\left[1-F\left(x_{i}\right)\right]^{R_{i}+2}\right] d x_{i} .
$$

Integration by parts leads to

$$
\begin{aligned}
I\left(x_{i+1}\right)= & \frac{x_{i+1}^{k+1}}{k+1}\left[1-F\left(x_{i+1}\right)\right]^{R_{i}+1}-\frac{x_{i-1}^{k+1}}{k+1}\left[1-F\left(x_{i-1}\right)\right]^{R_{i}+1} \\
& +\frac{R_{i}+1}{k+1} \int_{x_{i-1}}^{x_{i+1}} x_{i}^{k+1} f\left(x_{i}\right)\left[1-F\left(x_{i}\right)\right]^{R_{i}} d x_{i} \\
& +\frac{1-\alpha}{\alpha}\left[\frac{x_{i+1}^{k+1}}{k+1}\left[1-F\left(x_{i+1}\right)\right]^{R_{i}+2}-\frac{x_{i-1}^{k+1}}{k+1}\left[1-F\left(x_{i-1}\right)\right]^{R_{i}+2}\right] \\
& +\frac{1-\alpha}{\alpha} \frac{R_{i}+2}{k+1} \int_{x_{i-1}}^{x_{i+1}} x_{i}^{k+1} f\left(x_{i}\right)\left[1-F\left(x_{i}\right)\right]^{R_{i}+1} d x_{i} .
\end{aligned}
$$


Upon substituting Eq. (16) in Eq. (15) and re-arranging the resulting equation we get Eq. (14).

Corollary 2.3 For $\alpha=2$, we have

$$
\begin{aligned}
\mu_{i: m: n+1}^{\left(R_{1}, \ldots, R_{i}+1, \ldots, R_{m}\right)^{(k+1)}=} & \frac{2}{R_{i}+2} \frac{A(n+1, i-1)}{A(n, i-1)}\left[-(k+1) \mu_{i: m: n}^{\left(R_{1}, R_{2}, \ldots, R_{m}\right)^{(k)}}\right. \\
& -\left(n-R_{1}-\ldots-R_{i-1}-i+1\right) \mu_{i-1: m-1: n}^{\left(R_{1}, \ldots, R_{i-2}, R_{i-1}+R_{i}+1, R_{i+1}, \ldots, R_{m}\right)^{(k+1)}} \\
& +\left(R_{i}+1\right) \mu_{i: m: n}^{\left(R_{1}, R_{2}, \ldots, R_{m}\right)^{(k+1)}} \\
& +\left(n-R_{1}-\ldots-R_{i}-i\right) \mu_{i: m-1: n}^{\left(R_{1}, \ldots, R_{i-1}, R_{i}+R_{i+1}+1, R_{i+2}, \ldots, R_{m}\right)^{(k+1)}} \\
& -\frac{A(n, i)}{2 A(n+1, i-1)} \mu_{i: m-1: n+1}^{\left(R_{1}, \ldots, R_{i-1}, R_{i}+R_{i+1}+2, R_{i+2}, \ldots, R_{m}\right)^{(k+1)}} \\
& +\frac{A(n, i-1)}{2 A(n+1, i-2)} \mu_{i-1: m-1: n+1}^{\left.\left(R_{1}, \ldots, R_{i-2}, R_{i-1}+R_{i}+2, R_{i+1}, \ldots, R_{m}\right)^{(k+1)}\right] .}
\end{aligned}
$$

Corollary 2.4 For $\alpha=1$, we have

$$
\begin{aligned}
\mu_{i: m: n}^{\left(R_{1}, R_{2}, \ldots, R_{m}\right)^{(k+1)}=} & \frac{1}{R_{i}+1}\left[(k+1) \mu_{i: m: n}^{\left(R_{1}, R_{2}, \ldots, R_{m}\right)^{(k)}}\right. \\
& -\left(n-R_{1}-\ldots-R_{i}-i\right) \mu_{i: m-1: n}^{\left(R_{1}, \ldots, R_{i-1}, R_{i}+R_{i+1}+1, R_{i+2}, \ldots, R_{m}\right)^{(k+1)}} \\
& \left.+\left(n-R_{1}-\ldots-R_{i-1}-i+1\right) \mu_{i-1: m-1: n}^{\left(R_{1}, \ldots, R_{i-2}, R_{i-1}+R_{i}+1, R_{i+1}, \ldots, R_{m}\right)^{(k+1)}}\right] .
\end{aligned}
$$

Theorem 2.4 For $2 \leq m \leq n$ and $k \geq 0$ we have

$$
\begin{aligned}
\mu_{m: m: n+1}^{\left(R_{1}, \ldots, R_{m-1}, R_{m}+1\right)^{(k+1)}}= & \frac{\alpha A(n+1, m-1)}{(1-\alpha)\left(R_{m}+2\right) A(n, m-1)}\left[(k+1) \mu_{m: m: n}^{\left(R_{1}, R_{2}, \ldots, R_{m}\right)^{(k)}}\right. \\
& -\left(R_{m}+1\right) \mu_{m: m: n}^{\left(R_{1}, R_{2}, \ldots, R_{m}\right)^{(k+1)}} \\
& +\left(n-R_{1}-\ldots-R_{m-1}-m+1\right) \mu_{m-1: m-1: n}^{\left(R_{1}, \ldots, R_{m-2}, R_{m-1}+R_{m}+1\right)^{(k+1)}} \\
& \left.+\frac{1-\alpha}{\alpha} \frac{A(n, m-1)}{A(n+1, m-2)} \mu_{m-1: m-1: n+1}^{\left(R_{1}, \ldots, R_{m-2}, R_{m-1}+R_{m}+2\right)^{(k+1)}}\right], \\
\alpha> & 0, \alpha \neq 1 .
\end{aligned}
$$

Proof. Arguments similar to those in previous theorems, give us:

$$
\begin{aligned}
\mu_{m: m: n}^{\left(R_{1}, R_{2}, \ldots, R_{m}\right)^{(k)}}= & A(n, m-1)_{0<x_{1}<\ldots<x_{m-1}<\infty} I\left(x_{m}\right) f\left(x_{1}\right)\left[1-F\left(x_{1}\right)\right]^{R_{1}} \\
& \times \ldots f\left(x_{m-1}\right)\left[1-F\left(x_{m-1}\right)\right]^{R_{m-1}} d x_{1} \ldots d x_{m-1}
\end{aligned}
$$

where

$$
I\left(x_{m}\right)=\int_{x_{m-1}}^{\infty} x_{m}^{k}\left[1-F\left(x_{m}\right)\right]^{R_{m}+1} d x_{m}+\frac{1-\alpha}{\alpha} \int_{x_{m-1}}^{\infty} x_{m}^{k}\left[1-F\left(x_{m}\right)\right]^{R_{m}+1} d x_{m} .
$$


Integrating the last equation by parts, substituting the result in Eq. (18), we simply have

$$
\begin{aligned}
\mu_{m: m: n}^{\left(R_{1}, R_{2}, \ldots, R_{m}\right)^{(k)}=} & \frac{-1}{k+1}\left(n-R_{1}-\ldots-R_{m-1}-m+1\right) \mu_{m-1: m-1: n}^{\left(R_{1}, \ldots, R_{m-2}, R_{m-1}+R_{m}+1\right)^{(k+1)}} \\
& +\frac{R_{m}+1}{k+1} \mu_{m: m: n}^{\left(R_{1}, R_{2}, \ldots, R_{m}\right)^{(k+1)}} \\
& -\frac{(1-\alpha)}{(k+1) \alpha} \frac{A(n, m-1)}{A(n+1, m-2)} \mu_{m-1: m-1: n+1}^{\left(R_{1}, \ldots, R_{m-1}+R_{m}+2\right)^{(k+1)}} \\
& \left.+\frac{\left(R_{m}+2\right)(1-\alpha)}{(k+1) \alpha} \frac{A(n, m-1)}{A(n+1, m-1)} \mu_{m: m: n+1}^{\left(R_{1}, \ldots, R_{m-1}, R_{m}+1\right)^{(k+1)}}\right] .
\end{aligned}
$$

The result follows from Eq. (19).

Remark 2.1 One can use these derived relations to obtain the single moments of the progressive type-II right censored order statistics for some sample sizes and some censoring scheme $\left(R_{1}, \ldots, R_{m}\right), m \leq n$ in a simple recursive manner.

\section{$3 \quad$ Recurrence Relations for Product Moments}

In this section, we establish some recurrence relations for product moments of the progressive type-II right censored order statistics from the MOE distribution. The $(i, j)^{t h}$ product moment of the progressive type-II right censored order statistics can be written as

$$
\begin{aligned}
\mu_{i, j: m: n}^{\left(R_{1}, R_{2}, \ldots, R_{m}\right)}= & E\left[X_{i: m: n}^{\left(R_{1}, R_{2}, \ldots, R_{m}\right)} X_{j: m: n}^{\left(R_{1}, R_{2}, \ldots, R_{m}\right)}\right] \\
= & A(n, m-1)_{0<x_{1}<\ldots<x_{m}<\infty} x_{i} x_{j} f\left(x_{1}\right)\left[1-F\left(x_{1}\right)\right]^{R_{1}} f\left(x_{2}\right)\left[1-F\left(x_{2}\right)\right]^{R_{2}} \\
& \times \ldots f\left(x_{m}\right)\left[1-F\left(x_{m}\right)\right]^{R_{m}} d x_{1} \ldots d x_{m},
\end{aligned}
$$

where $f($.$) and F($.$) are given respectively in Eqs. (2) and (3) and A(n, m-1)$ is defined in Eq. (1). Then the product moments of the progressive type-II right censored order statistics given in Eq.(20) satisfy the following recurrence relations. 
Theorem 3.1 For $1 \leq i<j \leq m-1, m \leq n$ we have

$$
\begin{aligned}
\mu_{i, j: m: n+1}^{\left(R_{1}, \ldots, R_{j-1}, R_{j}+1, R_{j+1}, \ldots, R_{m}\right)=} & \frac{\alpha}{(1-\alpha)\left(R_{j}+2\right)} \frac{A(n+1, j-1)}{A(n, j-1)}\left[\mu_{i: m: n}^{\left(R_{1}, R_{2}, \ldots, R_{m}\right)}\right. \\
& -\left(R_{j}+1\right) \mu_{i, j: m: n}^{\left(R_{1}, R_{2}, \ldots, R_{m}\right)} \\
& +\left(n-R_{1}-\ldots-R_{j-1}-j+1\right) \mu_{i, j-1, m-1, n}^{\left(R_{1}, \ldots, R_{j-2}, R_{j-1}+R_{j}+1, R_{j+1}, \ldots, R_{m}\right)} \\
& -\left(n-R_{1}-\ldots-R_{j}-j\right) \mu_{i, j: m-1: n}^{\left(R_{1}, \ldots, R_{j-1}, R_{j}+R_{j+1}+1, R_{j+2}, \ldots, R_{m}\right)} \\
& -\frac{1-\alpha}{\alpha} \frac{A(n, j)}{A(n+1, j-1)} \mu_{i, j: m-1: n+1}^{\left(R_{1}, \ldots, R_{j-1}, R_{j}+R_{j+1}+2, R_{j+2}, \ldots, R_{m}\right)} \\
& \left.+\frac{1-\alpha}{\alpha} \frac{A(n, j-1)}{A(n+1, j-2)} \mu_{i, j-1: m-1: n+1}^{\left(R_{1}, \ldots, R_{j-2}, R_{j-1}+R_{j}+2, R_{j+1}, \ldots, R_{m}\right)}\right], \\
\alpha> & 0, \alpha \neq 1 .
\end{aligned}
$$

Proof. From Eq. (20) and Eq. (4) we have for $1 \leq i<j \leq m-1, m \leq n$ and $\alpha>0, \alpha \neq 1$

$$
\begin{aligned}
\mu_{i: m: n}^{\left(R_{1}, R_{2}, \ldots, R_{m}\right)}= & E\left[X_{i: m: n}^{\left(R_{1}, R_{2}, \ldots, R_{m}\right)}\left(X_{j: m: n}^{\left(R_{1}, R_{2}, \ldots, R_{m}\right)}\right)^{0}\right] \\
= & A(n, m-1)_{0<x_{1}<. .<x_{j-1}<x_{j+1}<\ldots<x_{m}<\infty} x_{i} L\left(x_{j}\right) f\left(x_{1}\right)\left[1-F\left(x_{1}\right)\right]^{R_{1}} \\
& \times \ldots f\left(x_{j-1}\right)\left[1-F\left(x_{j-1}\right)\right]^{R_{j-1}} f\left(x_{j+1}\right)\left[1-F\left(x_{j+1}\right)\right]^{R_{j+1}} \\
& \times \ldots f\left(x_{m}\right)\left[1-F\left(x_{m}\right)\right]^{R_{m}} d x_{1} \ldots d x_{j-1} d x_{j+1} \ldots d x_{m},
\end{aligned}
$$

where

$$
L\left(x_{j}\right)=\int_{x_{j-1}}^{x_{j+1}}\left[1-F\left(x_{j}\right)\right]^{R_{j}+1} d x_{j}+\frac{1-\alpha}{\alpha} \int_{x_{j-1}}^{x_{j+1}}\left[1-F\left(x_{j}\right)\right]^{R_{j}+2} d x_{j} .
$$


Integrating by parts Eq.(23) and substituting the result in Eq. (22), we obtain

$$
\begin{aligned}
\mu_{i: m: n}^{\left(R_{1}, R_{2}, \ldots, R_{m}\right)}= & \left(R_{j}+1\right) \mu_{i, j: m: n}^{\left(R_{1}, R_{2}, \ldots, R_{m}\right)} \\
& +\frac{(1-\alpha)}{\alpha}\left(R_{j}+2\right) \frac{A(n, j)}{A(n+1, j-1)} \mu_{i, j: m-1: n+1}^{\left(R_{1}, \ldots, R_{j}+1, \ldots, R_{m}\right)} \\
& -\left(n-R_{1}-\ldots-R_{j-1}-j+1\right) \mu_{i, j-1: m-1: n}^{\left(R_{1}, \ldots, R_{j-2}, R_{j-1}+R_{j}+1, R_{j+1}, \ldots, R_{m}\right)} \\
& +\left(n-R_{1}-\ldots-R_{j}-j\right) \mu_{i, j: m-1: n}^{\left(R_{1}, \ldots, R_{j-1}, R_{j}+R_{j+1}+1, R_{j+2}, \ldots, R_{m}\right)} \\
& +\frac{(1-\alpha)}{\alpha} \frac{A(n, j)}{A(n+1, j-1)} \mu_{i, j: m-1: n+1}^{\left(R_{1}, \ldots, R_{j-1}, R_{j}+R_{j+1}+2, R_{j+2}, \ldots, R_{m}\right)} \\
& -\frac{(1-\alpha)}{\alpha} \frac{A(n, j-1)}{A(n+1, j-2)} \mu_{i, j: m-1: n+1}^{\left(R_{1}, \ldots, R_{j-2}, R_{j-1}+R_{j}+2, R_{j+1}, \ldots, R_{m}\right)} .
\end{aligned}
$$

The result follows by re-arranging the last equation.

Corollary 3.1 For $\alpha=1$, we have

$$
\begin{aligned}
\mu_{i, j: m: n}^{\left(R_{1}, R_{2}, \ldots, R_{m}\right)}= & \frac{1}{R_{j}+1}\left[\mu_{i: m: n}^{\left(R_{1}, R_{2}, \ldots, R_{m}\right)}\right. \\
& -\left(n-R_{1}-\ldots-R_{j}-j\right) \mu_{i, j: m-1: n}^{\left(R_{1}, \ldots, R_{j-1}, R_{j}+R_{j+1}+1, R_{j+2}, \ldots, R_{m}\right)} \\
& \left.+\left(n-R_{1}-\ldots-R_{j-1}-j+1\right) \mu_{i, j-1: m-1: n}^{\left(R_{1}, \ldots, R_{j-2}, R_{j-1}+R_{j}+1, R_{j+1}, \ldots, R_{m}\right)}\right] .
\end{aligned}
$$

Corollary 3.2 For $\alpha=2$, we have

$$
\begin{aligned}
\mu_{i, j: m: n+1}^{\left(R_{1}, R_{2}, \ldots, R_{j}+1, \ldots, R_{m}\right)}= & \frac{2}{\left(R_{j}+2\right)} \frac{A(n+1, j-1)}{A(n, j-1)}\left[\left(R_{j}+1\right) \mu_{i, j: m: n}^{\left(R_{1}, R_{2}, \ldots, R_{m}\right)}-\mu_{i: m: n}^{\left(R_{1}, R_{2}, \ldots, R_{m}\right)}\right. \\
& -\left(n-R_{1}-\ldots-R_{j-1}-j+1\right) \mu_{i, j-1: m-1: n}^{\left(R_{1}, \ldots, R_{j-2}, R_{j-1}+R_{j}+1, R_{j+1}, \ldots, R_{m}\right)} \\
& +\left(n-R_{1}-\ldots-R_{j}-j\right) \mu_{i, j: m-1: n}^{\left(R_{1}, \ldots, R_{j-1}, R_{j}+R_{j+1}+1, R_{j+2}, \ldots, R_{m}\right)} \\
& -\frac{A(n, j)}{A(n+1, j-1)} \mu_{i, j: m-1: n+1}^{\left(R_{1}, \ldots, R_{j-1}, R_{j}+R_{j+1}+2, R_{j+2}, \ldots, R_{m}\right)} \\
& \left.+\frac{A(n, j-1)}{2 A(n+1, j-2)} \mu_{i, j-1: m-1: n+1}^{\left(R_{1}, \ldots, R_{j-2}, R_{j-1}+R_{j}+2, R_{j+1}, \ldots, R_{m}\right)}\right] .
\end{aligned}
$$


Theorem 3.2 For $1 \leq i \leq m-1, m \leq n$ and $\alpha>0, \alpha \neq 1$, we have

$$
\begin{aligned}
\mu_{i, m: m: n+1}^{\left(R_{1}, R_{2}, \ldots, R_{m}+1\right)}= & \frac{\alpha}{(1-\alpha)\left(R_{m}+2\right)} \frac{A(n+1, m-1)}{A(n, m-1)}\left[\mu_{i: m: n}^{\left(R_{1}, R_{2}, \ldots, R_{m}\right)}\right. \\
& -\left(R_{m}+1\right) \mu_{i, m: m: n}^{\left(R_{1}, R_{2}, \ldots, R_{m}\right)} \\
& +\left(n-R_{1}-\ldots-R_{m-1}-m+1\right) \mu_{i, m-1: m-1: n}^{\left(R_{1}, R_{2}, \ldots, R_{m-2}, R_{m-1}+R_{m}+1\right)} \\
& \left.+\frac{1-\alpha}{\alpha} \frac{A(n, m-1)}{A(n+1, m-2)} \mu_{i, m-1: m-1: n+1}^{\left(R_{1}, R_{2}, \ldots, R_{m-2}, R_{m-1}+R_{m}+2\right)}\right] .
\end{aligned}
$$

Proof. From Eq. (20) and upon using Eq. (4), we get for $1 \leq i \leq m-1$, $m \leq n$ and $\alpha>0$,

$$
\begin{aligned}
\mu_{i: m: n}^{\left(R_{1}, R_{2}, \ldots, R_{m}\right)}= & E\left[X_{i: m: n}^{\left(R_{1}, R_{2}, \ldots, R_{m}\right)}\left(X_{m: m: n}^{\left(R_{1}, R_{2}, \ldots, R_{m}\right)}\right)^{0}\right] \\
= & A(n, m-1)_{0<x_{1}<\ldots<x_{m-1}<\infty} x_{i} H\left(x_{m}\right) f\left(x_{1}\right)\left[1-F\left(x_{1}\right)\right]^{R_{1}} \\
& \times \ldots f\left(x_{m-1}\right)\left[1-F\left(x_{m-1}\right)\right]^{R_{m-1}} d x_{1} \ldots d x_{m-1},
\end{aligned}
$$

where

$$
H\left(x_{m}\right)=\int_{x_{m-1}}^{\infty}\left[1-F\left(x_{m}\right)\right]^{R_{m}+1} d x_{m}+\frac{1-\alpha}{\alpha} \int_{x_{m-1}}^{\infty}\left[1-F\left(x_{m}\right)\right]^{R_{m}+2} d x_{m} .
$$

The integration by parts of Eq. (26), leads to

$$
\begin{aligned}
I\left(x_{m}\right)= & -x_{m-1}\left[1-F\left(x_{m-1}\right)\right]^{R_{m}+1}-\frac{1-\alpha}{\alpha} x_{m-1}\left[1-F\left(x_{m-1}\right)\right]^{R_{m}+2} \\
& +\left(R_{m}+1\right) \int_{x_{m-1}}^{\infty} x_{m}\left[1-F\left(x_{m}\right)\right]^{R_{m}} d x_{m}+ \\
& \left(R_{m}+1\right) \frac{1-\alpha}{\alpha} \int_{x_{m-1}}^{\infty} x_{m}\left[1-F\left(x_{m}\right)\right]^{R_{m}+1} d x_{m}
\end{aligned}
$$

Upon substituting Eq. (27) in Eq. (25) and simplifying the result, we get

$$
\begin{aligned}
\mu_{i: m: n}^{\left(R_{1}, R_{2}, \ldots, R_{m}\right)}= & -\left(n-R_{1}-\ldots-R_{m-1}-m+1\right) \mu_{i, m-1: m-1: n}^{\left(R_{1}, R_{2}, \ldots, R_{m-1}+R_{m}+1\right)} \\
& +\left(R_{m}+1\right) \mu_{i, m: m: n}^{\left(R_{1}, R_{2}, \ldots, R_{m}\right)} \\
& -\frac{1-\alpha}{\alpha} \frac{A(n, m-1)}{A(n+1, m-2)} \mu_{i, m-1: m-1: n+1}^{\left(R_{1}, R_{2}, \ldots, R_{m-1}+R_{m}+2\right)} \\
& +\frac{1-\alpha}{\alpha}\left(R_{m}+2\right) \frac{A(n, m-1)}{A(n+1, m-1)} \mu_{i, m: m: n+1}^{\left(R_{1}, R_{2}, \ldots, R_{m}+1\right)}
\end{aligned}
$$

Obviously the result follows. 
Corollary 3.3 For $\alpha=1$, we have

$\mu_{i, m: m: n}^{\left(R_{1}, R_{2}, \ldots, R_{m}\right)}=\frac{1}{\left(R_{m}+1\right)}\left[\mu_{i: m: n}^{\left(R_{1}, R_{2}, \ldots, R_{m}\right)}+\left(n-R_{1}-\ldots-R_{m-1}-m+1\right) \mu_{i, m-1: m-1: n}^{\left(R_{1}, R_{2}, \ldots, R_{m-1}+R_{m}+1\right)}\right]$.

Corollary 3.4 For $\alpha=2$, we have

$$
\begin{aligned}
\mu_{i, m: m: n+1}^{\left(R_{1}, R_{2}, \ldots, R_{m}+1\right)}= & \frac{2}{\left(R_{m}+2\right)} \frac{A(n+1, m-1)}{A(n, m-1)}\left[-\mu_{i: m: n}^{\left(R_{1}, R_{2}, \ldots, R_{m}\right)}+\left(R_{m}+1\right) \mu_{i, m: m: n}^{\left(R_{1}, R_{2}, \ldots, R_{m}\right)}\right. \\
& -\left(n-R_{1}-\ldots-R_{m-1}-m+1\right) \mu_{i, m-1: m-1: n}^{\left(R_{1}, R_{2}, \ldots, R_{m-1}+R_{m}+1\right)} \\
& \left.+\frac{A(n, m-1)}{2 A(n+1, m-2)} \mu_{i, m-1: m-1: n+1}^{\left(R_{1}, R_{2}, \ldots, R_{m-1}+R_{m}+2\right)}\right] .
\end{aligned}
$$

Remark 3.1 Using these recurrence relations, one can obtain the product moments of the progressive type-II right censored order statistics for some sample sizes and some censoring schemes. Furthermore, it is possible to note that the recurrence relations for the standard exponential distribution and the half logistic distribution can be obtained as special cases when $\alpha=1$, and $\alpha=2$, respectively.

Remark 3.2 For the special case $R_{1}=R_{2}=\ldots=R_{m}=0$ so that $m=n$ in which the progressively censored order statistics become the usual order statistics $X_{1: n}, X_{2: n}, \ldots, X_{n: n}$ whose single and product moments are as defined in Salah, et al. [6], the recurrence relations established in Sections 2 and 3 are reduced to the following:

(a) From Eq. (8): For $k \geqslant 0$,

$$
\begin{aligned}
\mu_{1: n: n+1}^{(1,0, \ldots, 0)^{(k+1)}}= & \frac{(n+1) \alpha}{2 n(1-\alpha)}\left[(k+1) \mu_{1: n}^{(k)}-\mu_{1: n}^{(k+1)}-(n-1) \mu_{1: n-1: n}^{(1,0, \ldots, 0)^{(k+1)}}\right] \\
& -\frac{n-1}{2} \mu_{1: n-1: n+1}^{(2, \ldots, 0)(k+1)} .
\end{aligned}
$$

(b) From Eq. (14): For $2 \leq i \leq n-1$ and $k \geq 0$, we have

$$
\begin{aligned}
\mu_{i: n: n+1}^{(0, \ldots, 1, \ldots, 0)^{(k+1)}=} & \frac{\alpha}{1-\alpha} \frac{1}{2} \frac{A(n+1, i-1)}{A(n, i-1)}\left[(k+1) \mu_{i: n}^{(k)}\right. \\
& +(n-i+1) \mu_{i-1: n-1: n}^{(0, \ldots, 1, \ldots, 0)}{ }^{(k+1)} \\
& -\mu_{i: n}^{(k+1)}-(n-i) \mu_{i: n-1: n}^{(0, \ldots, 1, \ldots, 0)^{(k+1)}} \\
& -\frac{1-\alpha}{\alpha} \frac{A(n, i)}{A(n+1, i-1)} \mu_{i: n-1: n+1}^{(0, \ldots, 2, \ldots, 0)^{(k+1)}} \\
& \left.+\frac{1-\alpha}{\alpha} \frac{A(n, i-1)}{A(n+1, i-2)} \mu_{i-1: n-1: n+1}^{(0, \ldots, 2, \ldots, 0)^{(k+1)}}\right], \\
\alpha> & 0, \alpha \neq 1,
\end{aligned}
$$


while, in the superscript of the term on the left hand side, the 1 is in the $i-t h$ position, in the superscript of the second term on the right hand side, the 1 is in the $(i-1)-t h$ position, in the third term, however, 1 is in the $i-t h$ position, in the forth term, 2 is in $i-t h$ position, finally, in the fifth term, 2 is in the $(i-1)-t h$ position.

Remark 3.3 One can easily deduce some of the recurrence relations which derived in Salah, et, al. [6] from the recurrence relations given in the last two sections, for example when $R_{1}=R_{2}=\ldots=R_{m-1}=0$, so that there is no censoring before the time of the $m$ - th failure, then the first $m$ progressively type-II censored order statistics are simply the first $m$ usual order statistics. Based on such censoring scheme, we deduce the following: (1) From Eq. (8): For $n \geq 1, k \geq 0, \alpha>0$ and $\alpha \neq 1$

$$
\mu_{1: n+1}^{(k+1)}=\frac{\alpha}{2(1-\alpha)} \frac{n+1}{n}\left[(k+1) \mu_{1: n}^{(k)}-\mu_{1: n}^{(k+1)}-(n-1) \mu_{1: n}^{(k+1)}\right]-\frac{n-1}{2} \mu_{1: n+1}^{(k+1)} .
$$

Re-arranging Eq. (28) to get

$$
\mu_{1: n+1}^{(k+1)}=\frac{\alpha}{1-\alpha}\left[\frac{k+1}{n} \mu_{1: n}^{(k)}-\mu_{1: n}^{(k+1)}\right], n \geq 1, k \geq 0, \alpha>0, \alpha \neq 1 .
$$

This recurrence relation is equivalent to that established in Salah, et, al.[6], Theorem(1).

(2) From Eq. (14): For $2 \leq i \leq n-1, k \geq 0$ and $\alpha>0$, we have

$$
\begin{aligned}
(k+1) \mu_{i: n}^{(k)}= & \frac{1-\alpha}{\alpha} \frac{n-i+1}{n+1}\left[\frac{\alpha}{1-\alpha}(n+1) \mu_{i: n}^{(k+1)}-(n-i+2) \mu_{i-1: n+1}^{(k+1)}\right] \\
& +\frac{1-\alpha}{\alpha} \frac{(n-i+1)(n-i+2)}{n+1} \mu_{i: n+1}^{(k+1)}-(n-i+1) \mu_{i-1: n}^{(k+1)} .
\end{aligned}
$$

From Relation 3.3.1 see, Balakrishnan and Cohen [9], p. 24,

$$
i \mu_{i+1: n}^{(k)}+(n-i) \mu_{i: n}^{(k)}=n \mu_{i: n-1}^{(k)},
$$

we have the following two equations

$$
\begin{gathered}
\frac{\alpha}{1-\alpha}(n+1) \mu_{i: n}^{(k+1)}=\frac{\alpha}{1-\alpha} i \mu_{i+1: n+1}^{(k+1)}+\frac{\alpha}{1-\alpha}(n-i+1) \mu_{i: n+1}^{(k+1)}, \\
(n+1) \mu_{i-1: n}^{(k+1)}=(i-1) \mu_{i: n+1}^{(k+1)}+(n-i+2) \mu_{i-1: n+1}^{(k+1)} .
\end{gathered}
$$

By adding Eq. (30) to Eq. (31), we obtain

$$
\begin{aligned}
\frac{\alpha}{1-\alpha}(n+1) \mu_{i: n}^{(k+1)}+(n+1) \mu_{i-1: n}^{(k+1)}= & \frac{\alpha}{1-\alpha} i \mu_{i+1: n+1}^{(k+1)}+(n-i+2) \mu_{i-1: n+1}^{(k+1)} \\
& +\left[\frac{\alpha(n-i+1)}{1-\alpha}+i-1\right] \mu_{i: n+1}^{(k+1)} . \quad(32)
\end{aligned}
$$


Substituting Eq. (32) into Eq. (29), we simply have

$$
\begin{aligned}
\mu_{i+1: n+1}^{(k+1)}= & \frac{1}{i}\left[\frac{(n+1)(k+1)}{n-i+1} \mu_{i: n}^{(k)}-\frac{n-\alpha i+1}{\alpha} \mu_{i: n+1}^{(k+1)}\right. \\
& \left.+\frac{n+1}{\alpha} \mu_{i-1: n}^{(k+1)}\right], \quad 1 \leq i \leq n, n \geq 1, k \geq 0, \alpha>0 .
\end{aligned}
$$

This recurrence relation is equivalent to that established in Salah, et, al.[6], Theorem(2).

(3) From Eq. (21): The same arguments can be done as above to get

$$
\begin{gathered}
\mu_{i, j: n+1}=\mu_{i, j-1: n+1}+\left(\frac{\alpha}{1-\alpha}\right)\left(\frac{n+1}{n-j+2}\right)\left[\mu_{i, j-1: n}-\mu_{i, j: n}+\frac{1}{n-j+1} \mu_{i: n}\right], \\
1 \leq i<j \leq n, j-i \geq 2, \alpha>0, \alpha \neq 1 .
\end{gathered}
$$

This recurrence relation is equivalent to that established in Salah, et, al. [6], Theorem(5).

\section{Recursive Algorithm}

Using the recurrence relations established in Section 2 and 3 the means, variances and covariances of progressive type-II right censored order statistics from the standard MOE distribution can be readily computed as follows: Setting first $k=0$ and $n=1,2, \ldots$ in Eq. (11), to get

$$
\mu_{1: 1: n+1}^{(n)}=\frac{\alpha}{1-\alpha}\left[\frac{1}{n}-\mu_{1: 1: n}^{(n-1)}\right], \alpha>0, \alpha \neq 1 .
$$

Eq. (33) can be computed recursively by using

$$
\mu_{1: 1: 1}^{(0)}=-\frac{\alpha \ln \alpha}{1-\alpha}, \alpha>0, \alpha \neq 1
$$

Second, setting $k=1$ in Eq. (11), we obtain

$$
\mu_{1: 1: n+1}^{(n)^{(2)}}=\frac{\alpha}{1-\alpha}\left[\frac{2}{n} \mu_{1: 1: n}^{(n-1)}-\mu_{1: 1: n}^{(n-1)^{(2)}}\right], \alpha>0, \alpha \neq 1 .
$$

Also Eq. (34) can be done recursively by using Eq. (33) and with the help of

$$
\mu_{1: 1: 1}^{(0)(2)}=2 \alpha \sum_{j=1}^{\infty} \frac{(1-\alpha)^{j}}{(j+1)^{2}}
$$


Finally, one can obtain all the first and the second moments with $m=1$ for all sample sizes $n$. Using Eq. (8) with the help of Theorem 2.3 see, Balakrishnan and Aggarwala [11], p. 12, we can evaluate all moments of the form $\mu_{1 \cdot 2 \cdot n+1}^{\left(R_{1}+1, R_{2}\right)}$ for all $R_{1}, R_{2}, n=2,3, \ldots$, which can in turn be used, with Eq. (8) to determine all moments of the form $\mu_{1: 2: n+1}^{\left(R_{1}+1, R_{2}\right)^{(2)}}, n=2,3, \ldots$ Eq. (17) can be used to compute $\mu_{2: 2: n+1}^{\left(R_{1}, R_{2}+1\right)}$ for all $R_{1}, R_{2}$ and $n=2,3, \ldots$, these values can be used also to obtain all moments of the form $\mu_{2: 2: n+1}^{\left(R_{1}, R_{2}+1\right)^{(2)}}$. For $n \geq 2$ use Eq. (17) again. $\mu_{1: 3: n+1}^{\left(R_{1}+1, R_{2}, R_{3}\right)}$ and $\mu_{1: 3: n+1}^{\left(R_{1}+1, R_{2}, R_{3}\right)^{(2)}}$ can be computed from Eq. (8) for all $R_{1}, R_{2}, R_{3}$ and $n \geq 3$. Similarly for $\mu_{2: 3: n+1}^{\left(R_{1}, R_{2}+1, R_{3}\right)}$ and $\mu_{2: 3: n+1}^{\left(R_{1}, R_{2}+1, R_{3}\right)^{(2)}}$ can be determined from Eq. (14). Finally, $\mu_{3: 3: n+1}^{\left(R_{1}, R_{2}, R_{3}+1\right)}$ and $\mu_{3: 3: n+1}^{\left(R_{1}, R_{2}, R_{3}+1\right)^{(2)}}$ can be determined from Eq. (17). This process can be continued until all the desired first and second moments are obtained for all sample sizes and all censoring schemes.

The product moments of the form $\mu_{m-1, m: m: n+1}^{\left(R_{1}, \ldots, R_{m}+1\right)}, m=2,3, \ldots, n$ can be determined from Eq. (24). Also $\mu_{i-1, i: m: n+1}^{\left(R_{1}, \ldots, R_{m}+1\right)}, 2 \leq i \leq m$ can be computed from Eq. (24). Using Eq. (24) we can obtain some of the moments of the form $\mu_{m-2, m: m: n+1}^{\left(R_{1}, \ldots, R_{m}+1\right)}, m=3,4, \ldots, n$ and subsequently Eq. (21), moments of the forms $\mu_{i-2, i: m: n+1}^{\left(R_{1}, \ldots, R_{m}\right)}, 3 \leq i \leq m$ can be determined. Continuing this process, the desired product moments can be obtained.

\section{ACKNOWLEDGEMENTS:}

The Author would like to thank the Editor-in-Chief Prof. Belal Batiha and Prof. Mohammad Raqab for their valuable suggestions.

\section{References}

\section{References}

[1] A. W. Marshall and I. Olkin, A New Method for Adding a Parameter to a Family of Distributions with Application to the Exponential and Weibull Families, Biometrika 84 (1997) 641-652.

[2] B. Pradhan and D. Kundu, On Progressively Censored Generalized Exponential Distribution, Test 18 (2009) 497-515.

[3] K. S. Sultan, M. R. Mahmoud, and H. M. Saleh, Moments of Estimation from Progressively Censored Data of the Half Logistic Distribution, International Journal of Reliability and Applications 7(2) (2006) 187-201. 
[4] M. E. Ghitany, E. K. Al-Hussaini and R. A. A-Jarallah, Marshall-Olkin Extended Weibull Distribution and its Application to Censored Data, Journal of Applied Statistics 32(10) (2005) 1025-1034.

[5] M. M. Salah, On Marshall-Olkin Exponential Order Statistics and Associated Inferences, Ph.D Thesis, The University of Jordan, Amman, Jordan, 2010 .

[6] M. M. Salah, M. Z. Raqab and M. Ahsanullah, Marshall-Olkin Exponential Distribution: Moments of Order Statistics, Journal of Applied Statistical Science 17(1) (2008), 81-92.

[7] M. Z. Raqab, Generalized Exponential Distribution: Moments of Order Statistics, Statistics 38 (1) (2004) 29-41.

[8] N. Balakrishnan, Order Statistics from the Half Logistic Distribution, Journal of Statistical Computation and Simulation 20 (1985) 287-309.

[9] N. Balakrishnan and A. C. Cohen, Order Statistics and Inference: Estimation Methods, Academic Press, Boston,1991.

[10] N. Balakrishnan, E. Cramer, U. Kamps and N. Schenk, Progressive TypeII Censored Order Statistics from Exponential Distributions, Statistics 35 (2001) 537-556.

[11] N. Balakrishnan and R. Aggarwala, Progressive Censoring: Theory, Method and Applications, Birkhauser, Boston, 2000. 